\title{
La investigación espacial y su impacto en la sociedad hondureña
}

\begin{abstract}
CIENCIA Y TECNOLOGÍA (CT) Entrevista a la Doctora María Cristina Pineda, hondureña, Decana en funciones de la Facultad de Ciencias Espaciales. Es estudiosa de diversos temas de interés nacional e internacional, particularmente de los campos de Astronomía y Astrofísica, Ciencia y Tecnología de la Información Geográfica y Arqueoastronomía
\end{abstract}

\section{CT/ Desde que se inició el Observatorio Astronómico Centroamericano de Suyapa (OACS/UNAH) ¿cuáles son sus logros más importantes?}

MCP/ El haber conseguido poner en funcionamiento un observatorio astronómico en una universidad pública centroamericana ha sido un logro mundial grande, significativo y de suma importancia para la región. Más allá de esto, el haber creado las condiciones para que en nuevos campos del conocimiento, profesores y estudiantes podamos trabajar desde los ambientes académicos de la docencia, la investigación y la vinculación con la Sociedad que nos brinda la Universidad más grande y de más tradición en Honduras es otro logro importante. El hecho de estar estimulando que los habitantes de Honduras levantemos la vista al cielo para ver los planetas, las estrellas, las galaxias, a lo más profundo del cielo pero también a nuestro planeta para entender mejor su realidad y nuestro lugar en el Universo es un logro muy grande.

El haber creado las condiciones para que los niños y jóvenes de Honduras tengan ahora la opción de aprender a conocer mejor y a usar las Ciencias y las Tecnologías Espaciales, que las incorporen como parte de su diario quehacer y su cultura y que puedan aspirar a especializarse en la UNAH en los campos de la Astronomía y la Astrofísica, o de la Ciencia y las Tecnologías de la Información Geográfica, o de la Arqueoastronomía, son logros muy importantes. Hemos llegado a conformar un equipo de profesores, instructores, de personal administrativo y de servicio orgullosos de laborar en una de las unidades universitarias hondureñas de mayor prestigio nacional e internacional, que continuamente interactúa con profesores e investigadores que laboran en varios países y regiones del mundo. Hemos empezado a formar para Centroamérica, los primeros profesionales de postgrado de la Astronomía y la Astrofísica, y del Ordenamiento y la Gestión del Territorio. Y como un logro así mismo importante, el haber promovido que el Honorable Consejo Universitario de la UNAH, en reconocimiento al funcionamiento del OACS/UNAH creara la Facultad de Ciencias Espaciales. 


\section{CT/ ¿Cuál es la misión más importante del OACS/UNAH?}

MCPI La misión del OACS/UNAH ahora convertido en la Facultad de Ciencias Espaciales de la Universidad Nacional Autónoma de Honduras (FACES/UNAH), es la de contribuir al cumplimiento del mandato constitucional de organizar, dirigir y desarrollar la educación superior en Honduras, en los campos de las Ciencias Espaciales, específicamente en la Astronomía y la Astrofísica, la Ciencia y las Tecnologías de la Información Geográfica, la Arqueoastronomía, y otros campos afines. En este sentido nuestro ámbito de producción y acción es local, es regional, es universal. Nuestro compromiso es contribuir al desarrollo sostenible del país, por medio de la formación que brindamos a nuestros estudiantes, los productos de las investigaciones científicas que realizamos, y de las acciones de vinculación con la sociedad que promovemos, y por medio de la ciencia, la tecnología y la cultura que generamos, contribuir a que Honduras y el resto de los países centroamericanos, participemos de lo universal y nos desarrollemos en condiciones de equidad y humanismo.

\section{$\mathrm{CT} /$ ¿Qué tipo de investigaciones se realizan en el (OACS/UNAH?}

MCPI La conversión del OACS/UNAH en la Facultad de Ciencias Espaciales ha facilitado la organización de nuestros propios departamentos académicos, y con ello una mejor definición y distinción de las líneas de investigación científica en las que trabajan los profesores. Así por ejemplo, en Astronomía y Astrofísica se trabaja en Dinámica Planetaria: en la búsqueda de órbitas de planetas extrasolares y, en el estudio del efecto de la rotación de Venus debido al impacto de un anillo de gas en expansión; en Formación Estelar: en la detección de máseres en regiones de formación de estrellas de gran masa; en Cúmulos Galácticos: en la construcción de una base de espectros patrones en cúmulos estelares en las Nubes de Magallanes; en Instrumentación Astronómica: en la construcción de un radiotelescopio por ser este un rango espectral que ofrece mejores condiciones que el óptico para realizar observaciones astronómicas desde Honduras; en la Astronomía en la Cultura: estudiando la influencia de la Luna en los procesos agrícolas y en el tratamiento y explotación del bosque, así como la relación del desarrollo con el calentamiento global a partir del clima espacial en la región de Latinoamérica; en la Caracterización de las condiciones de observación del OACS/UNAH y en la Educación en Astronomía. En el campo de la Ciencia y las Tecnologías de la Información Geográfica se trabaja para formar parte de redes de: Laboratorios de Tecnologías de la Información Geográfica, de Infraestructuras de Datos Espaciales y de e-learning; se trabaja para poner en funcionamiento una Red Geodésica GPS para Tegucigalpa; en un Modelo de Realidad Virtual del Centro Histórico de Distrito 
Central; en Percepción Remota Cuantitativa: para la detección de cambios por cobertura y uso de la tierra en el occidente de Honduras; se trabaja en el Desarrollo de Nuevas Tecnologías para la protección y preservación del Patrimonio Cultural y Habitats Humanos en Honduras. En el campo de la Arqueoastronomía, se trabaja también en el Desarrollo de Nuevas Tecnologías para la preservación del Patrimonio Arqueológico y Hábitats Humanos desde esta nueva perspectiva; y se trabaja en laArqueoastronomía Maya.

\section{$\mathrm{CT} /$ ¿Qué impacto tienen las investigaciones que se realizan en el OACS para el pueblo hondureño?}

MCPI El impacto de las investigaciones que realizamos en el OACS se puede ver y medir en varias escalas. En la escala de los propios Departamentos Académicos de la Facultad de Ciencias Espaciales, que permite que cada uno de nuestros profesores se especialice en determinados campos que le son de su interés, dándole entonces con ello un sello personal y de prestigio al propio Departamento al que pertenecen. En la escala de la Universidad, al permitirnos posicionarnos como una unidad académica de alto nivel académico, reconocida por nuestra alta producción científica en relación al resto de las unidades académicas de la UNAH o de cualquier otra universidad o institución de educación superior del país. En la escala nacional, al conseguir que diferentes personas, organizaciones 0 instituciones nos señalen ahora como los especialistas para atender determinados campos del conocimiento para beneficio del desarrollo local, regional y del país. En la escala internacional, al estar incursionando con nuestras investigaciones en varios campos, estamos contribuyendo a darle al país y a la región centroamericana un lugar en un contexto donde hasta ahora habíamos sido los grandes ausentes, lo que nos está facilitando formar parte de grupos de investigación con reconocimiento en la Comunidad Científica Internacional.

\section{$\mathrm{CT} /$ ¿Existen relaciones con centros internacionales de investigación?}

MCPI Los campos del conocimiento en que trabajamos en el OACS solamente pueden ser desarrollados con propiedad si se trabajan de manera conjunta con grupos de investigadores activos laborando en diferentes lugares del mundo. De esta manera nuestras actividades académicas las desarrollamos por medio de proyectos de docencia, investigación, desarrollo e innovación que realizamos en colaboración con profesores que trabajan en grupos de investigación de departamentos, observatorios astronómicos y centros especializados de varias universidades de España, Argentina, Estados Unidos, México, Brasil y los países de Centroamérica. Para mencionar sólo algunos ejemplos, en Astronomía y 
Astrofísica, la acreditación regional que conseguimos para nuestro Programa de Maestría en Astronomía y Astrofísica, en parte se debió a que trabajamos con profesores de los observatorios de la Universidad Nacional de Córdoba, de la Universidad de La Plata y del Instituto de Astronomía y Física del Espacio de Argentina, de la Universidad Estadual Paulista de Brasil, de la Universidad Nacional Autónoma de México, de la Universidad de Cornell de Estados Unidos, de la Universidad Complutense de Madrid y de la Universidad de La Laguna de España, del Observatorio Astronómico del Vaticano.

Para poder abrir y poner en funcionamiento el campo de la Ciencia y las Tecnologías de la Información Geográfica, hemos trabajado con personal de la NASA y del Servicio Geológico de los Estados Unidos, del Departamento de Geografía de la Universidad de Alcalá de España, con quienes hemos logrado establecer relaciones con otras universidades españolas como la Universidad Politécnica de Madrid y la Universidad del País Vasco, con universidades latinoamericanas como la Universidad de Luján en Argentina, la Pontificia Universidad Católica de Chile y las universidades de Guadalajara y Sinaloa de México, o el Instituto Tecnológico de Cartago en Costa Rica. Para el desarrollo de la Arqueoastronomía nos hemos relacionado con investigadores de la Universidad de Colgate de Estados Unidos, la Universidad Politécnica de Madrid y la Universidad Politécnica de Barcelona de España. Como un hecho de enorme significado hemos logrado ingresar a la Unión Astronómica Internacional, organización que agrupa a más de diez mil astrónomos profesionales del mundo, teniendo entonces abiertas las puertas para interactuar, como ya lo estamos haciendo con la Comunidad Científica de unos ciento cincuenta países del mundo.

\section{CT/ ¿Qué investigaciones se han realizado en el área geoespacial y qué impacto han tenido en las mismas nuestras comunidades?}

MCPI Particularmente desde la Maestría en Ordenamiento y Gestión del Territorio, en la que ya tenemos los resultados de las primeras tesis, nuestros graduados han logrado incursionar en dos avenidas. Una, que tiene que ver con la regionalización de Honduras para la construcción de regiones plan a través del análisis espacial cuantitativo, siendo éste un trabajo de impacto nacional. La otra, que tiene que ver con el análisis multitemporal de la cobertura de la tierra en la Cuenca del Valle de Jesús de Otoro entre los años 2000 y 2006, para beneficio de los departamentos de Intibucá, La Paz y Comayagua. En el Departamento de Ciencia y Tecnologías de la Información Geográfica se ha logrado diseñar una metodología para la elaboración y mantenimiento de Sistemas de Información Geográfica (SIG) para la gestión de los centros históricos o sitios de valor patrimonial: aquí se completó el SIG del 
Distrito Central, cuyo diseño descansa en un escenario histórico que abarca la periodización de la conformación de las ciudades de Tegucigalpa y Comayagüela.

\section{CT/ Dentro del campo de Arqueoastronomía ¿nos puede indicar los logros alcanzados y su beneficio para Honduras?}

MCPI En el campo de la Arqueoastronomía los resultados los agrupamos en cuatro grandes categorías. La primera, que recoge los resultados cuando descubrimos numerosos alineamientos de las estelas, graderías y estructuras del sector norte de la Plaza Principal del Parque Arqueológico de Copán Ruinas, asociadas con las salidas o puestas del sol en las fechas de eventos solares importantes tales como equinoccios, solsticios y pasos del Sol por el cenit en Copán. La segunda, cuando al ubicar en la línea del tiempo los resultados anteriores, pudimos develar el Grande y Complejo Plan del Gobernante 18 Conejo para la construcción del sector norte de la Plaza Principal de Copán Ruinas, al que propusimos llamar Plaza del Sol. La tercera cuando logramos demostrar como pudieron haber sido utilizadas las estelas de la Plaza del Sol como instrumentos para medir la posición del Sol sobre el horizonte en las fechas de eventos solares de interés, y a partir de ello medir la duración del año trópico llamado por los mayas el haab. Y la cuarta, cuando descubrimos que la Estela $D$ y su altar, junto con las graderías que la rodean en el sector norte de la Plaza del Sol funcionaba como un reloj solar que seguramente el Gobernante 18 Conejo mandó a construir para marcar las divisiones del día y la duración del año trópico. Desde luego todos estos resultados contribuyen a entender mejor los conocimientos astronómicos que tenían y usaban los mayas, para el desarrollo de sus sociedades, lo cual es de enorme beneficio para nuestro país.

\section{CT/ ¿Qué nuevas disciplinas científicas se han auspiciado dentro del OACS?}

MCPI El Observatorio Astronómico inicialmente se creó para poner en funcionamiento el campo de la Astronomía y la Astrofísica que antes de la década de los noventa no se ofrecía en la UNAH, ni en ninguna otra universidad o centro de educación superior del país. En este campo, desde entonces, trabajamos en las disciplinas de: Sistema Solar, El Sol, Estrellas, Galaxias, Instrumentación Astrofísica y Educación en la Astronomía. Al integrarnos a equipos internacionales de investigación, pronto empezamos a conocer el campo de las Ciencias Espaciales, y los enormes beneficios que para el desarrollo social y económico de los países de ellas se derivaban, por lo que casi inmediatamente empezamos a trabajar en sus diferentes disciplinas, contribuyendo desde el OACS a la construcción de capacidades en educación e investigación en este campo, para beneficio de nuestro 


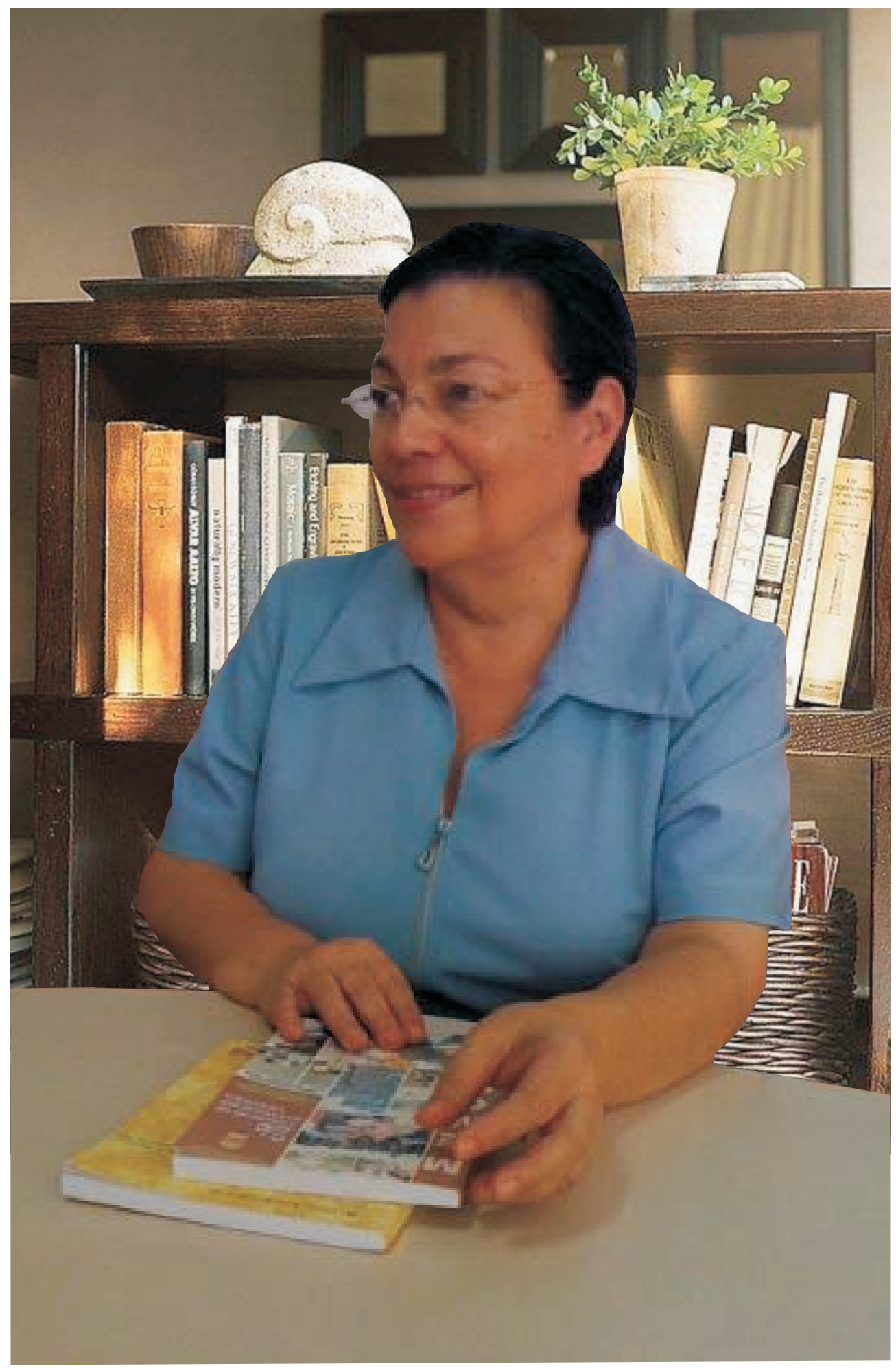


país y nuestra región. Fue así como empezamos a trabajar en la Percepción Remota primero, por cierto, en un proyecto regional con la NASA; luego, con los Sistemas de Información Geográfica, con la Fotogrametría, la Cartografía Espacial, los Sistemas de Geoposicionamiento Global, hasta llegar a abarcar casi completamente el campo de la Ciencia y las Tecnologías de la Información Geográfica.

El campo de la Arqueoastronomía surgió derivado de la Maestría en Astronomía y Astrofísica, desde donde inicialmente nos propusimos estudiar cuáles eran los conocimientos astronómicos de los Mayas, particularmente los que habitaron en el occidente de Honduras. De esta manera empezamos a trabajar en la Arqueoastronomía Maya primero, pero luego, y por trabajar en las Ciencias Espaciales, recientemente hemos empezado a trabajar en las Nuevas Tecnologías para la Preservación del Patrimonio Nacional.

\section{CT/ ¿Qué condiciones de observación astronómica tiene el OACS?}

MCP/Para explicar las condiciones de observación astronómica tenemos que hacer referencia, por un lado, a las condiciones según el rango del espectro electromagnético al que podamos acceder, y por otro, a los lugares desde donde podemos hacer las observaciones astronómicas; esto, dado el carácter del OACS de ser una unidad académica universitaria. En cuanto al rango del espectro electromagnético y por el tipo de telescopio que tenemos instalado en la cúpula, tenemos una capacidad para trabajar en horas nocturnas y de las madrugadas en el rango óptico; este rango tiene las limitaciones de que cuando hay nubes o está lloviendo no se pueden hacer observaciones, lo cual ocurre en Tegucigalpa con más frecuencia entre los meses de mayo a noviembre. Sin embargo, y para ampliar el número de días de observación, ya estamos haciendo estudios e investigaciones para construir nuestro propio radiotelescopio, lo que nos permitirá hacer observaciones a cualquier hora del día y en cualquier fecha del año, haciendo desde luego las observaciones en la región de radio del espectro electromagnético.

En cuanto al lugar desde donde podemos hacer observaciones, las podemos hacer utilizando el Telescopio "Rene Sagastume Castillo" que tenemos instalado en la cúpula de la Ciudad Universitaria de Tegucigalpa (Latitud: $14^{\circ} 05^{\prime}$ Norte; Longitud: $87^{\circ}$ 09' Oeste; Altura: 1077 metros sobre el nivel del mar), o desde los predios alrededor de la cúpula utilizando pequeños telescopios portátiles. Por el tipo de tecnologías a las que ahora podemos acceder, adicionalmente, desde nuestras aulas y oficinas de trabajo en la Ciudad Universitaria de Tegucigalpa, hemos empezado a operar y a utilizar de manera virtual varios radio telescopios que físicamente están instalados en otros países e inclusive en otros continentes, lo cual 
nos ha abierto posibilidades enormes para realizar nuestras observaciones e investigaciones desde el OACS/UNAH.

\section{CT/ ¿Cuál es el enfoque que tiene OACS del calentamiento global?}

MCP/El calentamiento global es un gran problema para toda la humanidad, el cual debe ser estudiado seriamente y exhaustivamente para buscarle soluciones desde todas las perspectivas posibles. En el OACS lo abordamos desde varios puntos de vista, según el campo en que trabajan nuestros profesores. Por el lado de la Ciencia y las Tecnologías de la Información Geográfica, lo abordamos estudiando las características de la superficie de la tierra y los cambios asociados que se pueden detectar por análisis multitemporales, cambios que pueden estar asociados con variaciones estacionales o con variaciones de una mayor pervivencia, cambios asociados a catástrofes naturales o a alteraciones de origen humano.

Por el lado de la Astronomía y la Astrofísica, lo hemos empezado a abordar desde el estudio de la actividad solar y su influencia en el clima de la tierra, y desde el estudio del clima espacial. Por el lado de la Arqueoastronomía lo estudiamos, conociendo mejor las características propias de la zona geográfica en que vivimos, las formas en que desde las primeras culturas que habitaron nuestro territorio las percibieron hasta como lo hacemos nosotros, y esto para distinguirlas de otro tipo de características que puedan tener un origen global.

\section{CT/ ¿Qué relación existe entre los trabajos del OACS/UNAH y la Dirección de Investigación Científica?}

MCPI Desde su puesta en funcionamiento el OACS nació como una unidad universitaria donde la investigación científica era parte fundamental del quehacer de sus profesores y estudiantes. Ningún profesor universitario nuestro puede tener una Carga Académica que no incluya el desarrollo o la participación en algún proyecto de investigación científica. Nuestra relación con la Dirección de Investigación Científica, se ha empezado a estrechar particularmente en los últimos años, cuando su actual Dirección, a raíz de los procesos de reforma universitaria de la UNAH y del apoyo que ha recibido de las autoridades universitarias, está consiguiendo crear las bases y condiciones para cumplir sus funciones de dirigir los procesos académicos de investigación científica en todos sus aspectos.

Con la Dirección de Investigación Científica en los años más recientes, por ejemplo, hemos trabajado en la definición de los procedimientos para el desarrollo de los proyectos de investigación científica en nuestros departamentos académicos; 
nuestros profesores han recibido ayuda para perfeccionar las formas de presentar sus diseños metológicos como requisito previo para registrar sus proyectos; los profesores nos hemos visto estimulados a competir para conseguir becas de apoyo al desarrollo de los proyectos de investigación científica que queremos realizar; el personal de la Dirección de Investigación Científica nos ha impulsado a perfeccionar las formas de presentar públicamente los resultados de nuestras investigaciones, particularmente valorando la importancia y la forma de publicar nuestros resultados a través de la propia revista científica especializada.

Estamos claros que en materia de investigación científica hay mucho por hacer, pero tenemos la confianza que acompañados por el equipo de la Dirección de Investigación Científica vamos a llegar más lejos, sobre todo a partir de ahora que ya hemos conformado la Unidad de Investigación Científica de la Facultad de Ciencias Espaciales.

\section{CT/ ¿Podría explicarnos qué líneas de investigación tienen para el futuro inmediato dentro del OACS?}

MCPI Los retos del futuro inmediato del OACS están condicionados por el apoyo que las autoridades universitarias nos brinden para organizar, en todos sus aspectos, la Facultad de Ciencias Espaciales (FACES) en la que ahora nos hemos convertido. Funcionamos de hecho con una nueva estructura organizativa, nos esforzamos por trabajar en los nuevos campos que hemos abierto, pero será sólo hasta cuando oficialmente nos aprueben los nuevos puestos que nuestra nueva unidad académica demanda, que nos permitan nombrar y con ello disponer de nuestro propio personal que podremos alcanzar mayores resultados.

Seguiremos desde luego participando con los equipos internacionales que el desarrollo de nuestros proyectos y actividades requieren, ahora desde los campos científicos mejor definidos y por los que hemos luchado para que funcionen en la UNAH y en el país, pero lo tenemos bien claro, si no contamos con el apoyo institucional de manera evidente y directa, nuestros esfuerzos tendrán que ser extremos. Somos una nueva Facultad, pero aún no podemos participar conforme ley, en los organismos de gobierno universitario; no se nos permite participar directamente con la administración académica de la Universidad.

Aun cuando tengamos altos reconocimientos internacionales, pasamos por una situación interna difícil. Confiamos en que, cuando las autoridades universitarias tengan más claro el enorme potencial de la Facultad de Ciencias Espaciales, ellas mismas nos abrirán los espacios que nos permitirán realizar plenamente nuestro 
trabajo.

En ese momento las líneas de investigación que estamos impulsando desde las Astronomía y la Astrofísica, la Ciencia y las Tecnologías de la Información Geográfica y la Arqueoastronomía, tendrán un mayor impacto para nuestros estudiantes universitarios, para los niños, para los jóvenes, para los hondureños y los centroamericanos en general. 